\title{
La investigación en los posgrados académicos y profesionalizantes en Honduras
}

\section{Research in academic and professional postgraduate programsin Honduras}

\author{
Edwin Roldan Medina López \\ Investigador Residente de Posgrado UPN FM \\ edwinhts@yahoo.com
}

\section{Resumen}

En Honduras no existe actualmente una diferenciación entre posgrados académicos y profesionalizantes, por lo que la formación que se brinda para el trabajo académico termina considerándose equivalente a la formación para el trabajo profesional y viceversa. Por otro lado, los trabajos de investigación del posgrado académico se transforman en el modelo de investigación a seguir también para los posgrados profesionales. Esto lleva a plantear problemas en la educación de posgrado dado que no se estimula, e incluso se desmo tiva, la formulación de propuestas de buena calidad para la formación profesional en aras de una formulación de propuestas con formato académico que en el mejor de los casos inhibe la investigación y en el peor de los casos crea híbridos que no son ni buenos profesionales ni buenos investigadores. Se propone profundizar en esta diferenciación y elaborar a partir del análisis resultante, propuestas de programas de posgrado congruentes con las necesidades y realidades de la formación que se 
Edwin Roldán M edina López

brinda en el nivel educativo superior, particularmente en el nivel de posgrado que contribuyan al desarrollo del país.

Palabras clave: Investigación/ Programas de posgrado/Tesis/ Metodología de Investigación

\begin{abstract}
Abstrac
In Honduras, currently there is no difference between academic and professional postgraduate programs. That is why the formation given for academic work ends up being considered equivalent to that of the professional work and viceversa. Research work for academic postgraduate programs, on the other hand, becomes the research model to be followed by professional postgraduate programs as well. This leads to problems in the education at the postgraduate level since it does not promote, and even discourages, the making of good quality proposals for professional areas in the pursuit of making proposals with a more academic format, which in the best case scenario inhibits research and in the worst case it creates hybrids that are neither good professionals nor good researchers. The proposal is to dig deeper into this difference and from the resulting analysis, to elaborate postgraduate programs in accordance with the needs and reality of the formation given at the higher educational level, particularly in the postgraduate programs so that they can contribute to the development of the country.
\end{abstract}

Keywords: Research/Postgraduate programs/ Thesis/Research methodology

\title{
Introducción
}

Los posgrados en Honduras han tenido un incremento exponencial a partir del año 2000 (A maya, 2014); esto concuerda con la expansión generalizada de los posgrados a nivel mundial que ha sido el instrumento mediante el cual el mundo univer sitario está respondiendo a las demandas de la era del conocimiento a través de la oferta de 
saber es especializados. (Rama, 2007).

Las áreas privilegiadas con la apertura de los posgrados y las que cuentan con mayor número actualmente por área de formación son: salud, educación y ciencias económicas aunque también la oferta incluye áreas como ciencias jurídicas, ciencias sociales, ingenierías, ciencias y letras. En el año 2008 se contaba con 64 programas de posgrado en la UN AH, a la fecha se ofertan 85 diferentes programas con maestrías en áreas novedosas en el país como la especialidad en medicina Legal y forense y la Maestría en Estado y Ciencias Políticas. (UN AH, 2008,2014)

En la Universidad Pedagó gica N acional Francisco Morazán (UPN FM) la situación es muy similar y a partir del año 2000 se ha incrementado el número de maestrías orientadas en su mayoría al área de educación y se cuenta además con dos doctorados en educación.

Al analizar los procesos y requerimientos de graduación en prácticamente todos los programas de posgrado se establece la Tesis de Grado como conditio sine qua non para obtener la titulación. La realización de esta tesis implica la elaboración de un trabajo de investigación que independientemente del tipo u orientación del posgrado debe cumplir con los requisitos de un trabajo académico, en detrimento de los posgrados con una orientación profesionalizante.

En el presente trabajo se plantea no solamente la necesidad de diferenciar ambos tipos de posgrado, sino también la necesidad de diferenciar el rol que juega o debería jugar la investigación en cada uno de ellos. El documento se estructura en varios apartados que buscan mostrar que la diferencia entre ambos tipos de posgrado va más allá de una diferencia meramente de nombre. Se parte del origen y situación actual de los posgrados; se propone una diferencia entre un posgrado académico y uno profesionalizante, se analiza el componente de investigación en los planes de estudio actuales, se analiza cómo y que se enseña sobre metodología de investigación, sobre la diferencia en lo s trabajo s finales y la aseso ría académica y se plantea además el campo profesional en que se pueden desarrollar ambos tipos de profesionales. 
Edwin Roldán M edina López

\section{Metodosy materiales}

El presente trabajo se desarrolló mediante una investigación desde el enfo que cualitativo de tipo documental y con un alcance argumentativo exploratorio (Montemayor, García y Garza, 2002) basada en fuentes primarias, artículos e informes de investigación, sobre la temática de los posgrados académicos y profesionalizantes en la que se realiza un análisis, con el propósito de establecer relaciones, diferencias, etapas, posiciones y el estado actual del conocimiento respecto al tema. En este sentido se establecieron categorías de análisis para establecer las diferencias entre ambos tipos de posgrado como ser:a) La investigación en los planes de estudio, b) La meto dología de investigación, c) El trabajo final de posgrado y la asesoría y d) el campo profesional.

\section{DiscusiónTeórica}

\section{Origen y Situación Actual de los Posgrados}

Según estudios realizados por Jorge A maya (2014) los antecedentes más remotos de la promoción de estudios a nivel de Postgrado en H onduras se remontan a la primera reforma que sufrió la U niversidad Central a fines del siglo XIX, concretamente en 1882 durante el gobierno de la Reforma Liberal de Marco Aurelio Soto. La reforma educativa, guiada por Ramón Rosa, impulsó la instauración del paradigma de la filosofía positivista, que promovía la formación de cuadros científicos y técnicos, a diferencia del modelo escolástico precedente. La universidad entonces y a partir de la iniciativa del entonces rector $D$ r.A do Ifo Zúniga, fue dividida en facultades, contando con cuatro facultades: Jurisprudencia, Ciencias Políticas, Medicina y Cirugía y C iencias. La nueva organización universitaria estableció que al término de los estudios se otorgarían los títulos de licenciado y también se reglamentó el sistema para la obtención de doctorados, que en ese tiempo se ofrecían únicamente doctorados en Derecho. (Amaya, 2014).

Los estudios de posgrado como los conocemos ahora, fueron 
impulsados por la UN AH a partir de los años 1960 en el proceso de la II Reforma Universitaria que organizó los estudios generales en la UNAH a través de la creación del Centro Universitario de Estudios Generales (CUEG). Aunado a este proceso hubo otros factores que promovieron la apertura de programas de posgrado en diferentes áreas. Estos factores incluyeron la promoción de becas para estudiar posgrados en el país (y fuera del mismo) y la contratación sistemática de profesores de alto nivel sobre to do de Españay de algunos exiliados suramericanos entre los que destacan A ugusto Serrano, A tanasio Herranz, Clodomir Santos de Morais, Héctor Palacios, Héctor Pérez Brignoli,Juan A rancibia, C arlosVilas, Franz H inkelamert,Vin D ierxchens, entre otros. (A maya, 2014) .

En las épocas de los 70 y 80 s se impulsaron en la UN AH posgrados en las áreas de medicina, ciencias sociales y economía, sobresaliendo entre ellos el primer postgrado de la UNAH acreditado a nivel latinoamericano, el posgrado en Trabajo Social, Se crea también el Posgrado Centroamericano en Economía (PO SCAE) y la maestría en Población y Desarrollo. Estos programas siguen funcionando en la actualidad a excepción de la maestría en población y desarrollo que entró en un proceso de desgate y no continuó funcionando (Amaya, 2014). Entre el año 2000 y el 2005 los programas de posgrado se incrementaron en alrededor de un 112 por ciento y entre el 2008 y el 2014 el incremento ha sido de alrededor de un $25 \%$ pasando de 64 posgrados a 85 autorizados en el 2014.

En la UPN FM, los posgrados surgen en el año de 1997 con la apertura de la maestría en Curriculum, administrada por la Dirección de Posgrado, creada en el año de 1996 con el fin de promover, dirigir y coordinar los programas de posgrado.A la fecha la U PN FM ofrece 14 programas de maestría y dos Doctorados todos orientados en educación, y que buscan capacitar profesionales e investigadores que puedan incidir en los procesos y en las políticas públicas en aspectos relacionados con la educación, particularmente en los niveles prebásico, básico y medio. Entre el año 1997 y 2001 se abrieron tres programas de maestría: Curriculum, Enseñanza de la Geografía y 
Educación, en el año 2000 se abrieron cuatro programas Estudios de Género y Educación, Investigación Educativa, Matemáticas y Educación Física y el resto se aperturaron a partir del 2004. La Maestría de más reciente apertura (2014) es la de Gestión Administrativa y Financiera y próximamente se inaugurará la primera promoción de la maestría en Bibliotecología. (UPN FM, 2014) .

Como se puede apreciar, la tendencia es a incrementar la oferta de estudios a nivel de posgrado, los requerimientos actuales de apertura de los nuevos posgrados incluyen el estudio de las necesidades del país desde una visión regional, lo que plantea la expansión la de la oferta hacia áreas y sectores que no habían contado con esta posibilidad y que cuentan con características y potencialidades que no se han explotado plenamente, como ejemplo se cita el caso de la maestría en C iencias A cuícolas en Choluteca y la de Manejo de Recursos C osteros en La C eiba y la maestría en Bibliotecología en Tegucigalpa.

\section{Diferencia entre PosgradosAcadémicos y Profesionalizantes}

En Honduras la reglamentación vigente, aunque en proceso de reformulación, aún no considera las características diferenciales entre ambos tipos de posgrados. El énfasis está puesto en los criterios académicos por lo que terminan siendo perjudicados, por los resultados de la evaluación, la acreditación y la eficiencia, los posgrados profesionalizantes.

Lo que esto significa es que si los posgrados se deben evaluar y acreditar en función de la eficiencia terminal que logran, en el país por esta razón se tienen grandes dificultades para lograr la acreditación de los programas de posgrado, de tal manera que solamente la Maestría en D emografía Social de la UNAH está actualmente acreditada a nivel centroamericano por parte de laACAP.

El porcentaje de eficiencia terminal en la mayoría de programas de posgrado es de alrededor del 30 por ciento; so lamente las especialidades médicas escapan a esta realidad ya que cuentan con una eficiencia ter- 
minal del 99 por ciento, claramente el formato y la estrategia en que se ofrecen estas especialidades parece hacer la diferencia.

Volviendo a la idea inicial, a pesar de las diferencias claras entre los diferentes programas y áreas de especialización, los criterios académicos establecidos de manera genérica - para todos los programas y todas las universidades- por el sistema de Educación Superior del país, enfatizan en la realización de un proceso de investigación para el cual los estudiantes en muchos casos no están lo suficientemente preparados, más adelante se analizará por qué. En tanto, es importante tratar de establecer una diferencia al menos conceptual entre ambos tipos de programas.

Según la Dirección de Investigación de Postgrado de la UNAH la diferencia radica principalmente en el enfoque o tipo de investigación que se realiza en cada programa, es decir; una maestría académica es un proceso de formación fundamentado en la realización de investigación básica, en tanto que las maestrías profesionalizantes se fundamentan en procesos de investigación aplicada. (D IC yP, 2014)

\section{La Metodología de Investigación en los Posgrados}

Un breve análisis sobre la naturaleza de los programas de posgrado llevaría a concluir que al ser los programas y el enfoque de la investigación diferentes en ambos tipos de posgrado, la enseñanza de la metodología de investigación, también debería ser diferente, pero este no es el caso en los posgrados en el país. La metodología de investigación se enseña a todos y en todos los posgrados por igual, de manera teórica y retórica, con muy poca oportunidad para la práctica y salvo casos particulares, con muy poco apego a la problemática del área específica.

Por lo general en los posgrados se enseña basándose en un libro de texto de Hernández, Fernández y Baptista, conocido popularmente como el Libro de Sampieri o la biblia de la investigación. En el pregrado se usa el libro de Cesar Bernal. El libro dicta lo que se debe de enseñar 
y cómo hacerlo. Un libro, una forma de hacer las cosas. Eso de alguna manera, al menos en teoría, facilitaría la realización de trabajos de investigación pues todos seguirían el mismo esquema, la misma metodología y llegarían al mismo resultado, la tesis. Pero, ¿Q ué pasa cuando no se necesita hacer una tesis sino un trabajo práctico como en el caso de las maestrías Profesionalizantes? 0 ¿Q ué pasa si se les enseña cómo hacer una tesis solo teóricamente y luego se pide a los estudiantes un producto con todos los "requerimientos" técnicos y metodo ló gicos que exige el posgrado? La baja eficiencia terminal podría ser una buena respuesta aunque quizás no la única pues además de la problemática de las debilidades planteadas en cuanto a la enseñanza y aprendizaje de la meto do lo gía de investigación, hay otros aspectos que inciden en la realización de un adecuado trabajo en función del tipo de posgrado del que se trate, académico o profesionalizante.

\section{LosTrabajos Finales y la Asesoría Académica}

Tener claridad en el tipo de posgrado es el primer paso para ir eventualmente (re) ajustando los procesos que permitan llegar a propuestas de investigación que vayan más allá de la actual y férrea tesis. Es decir, un profesional o especialista en un área específica debería ser capaz de plantear soluciones o alternativas a problemas "reales" de la sociedad, la empresa o el estado; estas soluciones pueden tener la forma de diagnósticos, estudios de caso, estudios de mercado, proyectos, sistematizaciones, investigación acción, evaluaciones de impacto, entre otras. Pero para ello, y aquí otro aspecto a considerar en la diferenciación de ambos tipos de programa, las maestrías profesionalizantes deberían estar vinculadas con la sociedad civil o rganizada, una institución, empresa u organismo que pueda plantearles sus necesidades y que ellos desde su área de expertise y de los cono cimientos obtenidos en sus estudios de posgrado puedan aportar a la solución de los problemas que les acontecen. Es decir que los programas de posgrado profesionalizante no deberían estar orientados a la comunidad, sino basados en la comunidad.

0 tro problema que se encuentra en ambos tipos de programa es el 
papel del asesor o tutor de tesis. Los asesores hacen lo que alguien más hizo anteriormente con ellos al mo mento de asesorarles, es decir, y volviendo al caso de Enseñanza de la Matemática (UPN FM) y de Derecho marítimo (UNAH) que los graduados de estos programas, ambos profesionalizantes, se convierten en asesores de una tesis académica; las debilidades y fortalezas que ellos mismos enco ntraron en su proceso de formación se siguen repitiendo, pues el profesor y el abo gado no son investigadores y de pronto se ven enfrentados a apoyar en un proceso de investigación en su área profesional, pero del cual su única experiencia (¿traumática?) fue su tesis de grado. No existen procesos de certificación de asesores de tesis y tampoco existe un reconocimiento profesional por esta labor, así quien decide ser asesor de tesis en muchos casos debe (re)aprender a investigar junto con el estudiante al que asesora. Por otro lado, se cuenta con investigadores experimentados y personas que manejan muy bien los aspectos metodológicos y técnicos del proceso de investigación, pero no conocen las diferentes áreas en las que se les solicita su apoyo, eventualmente, ante la falta de asesores se vuelven "todólogos" que aseso ran tesis tanto de matemáticas como de ciencias naturales o de derechos humanos y recursos hídricos. Esto, obviamente, plantea la duda sobre la calidad metodológica de una tesis asesorada por un experto y la calidad teórica de una tesis asesorada por un meto dólogo. A nte esta problemática se ha desarrollado recientemente un modelo en el que se plantea tener dos asesores de tesis, uno para el área metodológica y otro para el área específica de profesionalización.

Lo anteriormente planteado solo puede suceder en una maestría profesio nalizante, en una maestría académica, los docentes que imparten las clases son investigadores que conocen la temática y conocen la meto dología de investigación en esa temática y hacen del proceso de aprendizaje práctico y acompañan al estudiante en todo el camino, desde el inicio hasta el final de su trabajo de investigación (Algo como lo que sucede en los posgrados de medicina aunque estos no son académicos sino profesionalizantes), haciéndole partícipe de investigaciones reales en diferentes momentos del proceso, a veces como recolector de datos o muestras, a veces como analista, a veces 
Edwin Roldán M edina López

como escritor, a veces como presentador de resultados. En algunos países incluso existe la figura del asistente del profesor, 0 asistente del investigador, quien durante su proceso de formación, además de las responsabilidades académicas propias del posgrado, asiste al docente que imparte clases y de esta manera va adquiriendo conocimientos y habilidades para las que el aula de clase no es suficiente.

\section{El Campo Profesional}

Según Sánchez (2008) el graduado de maestrías académicas podrá incorporarse al ámbito laboral como un investigador docente y el graduado de las maestrías profesionalizantes podrá laborar como un profesional docente. Este planteamiento en el que ambos graduados se coinvierten en docentes, lleva a pensar que el autor está pensando en las instituciones de educación superior como el espacio en el que se desarrollarán los graduados de ambos tipos de programa. La universidad puede ser vista como un área de trabajo, pero no como la única y particularmente si se trata de un posgrado profesionalizante.

El campo profesional, siendo un poco simplistas si se quiere, es en el área de investigación para los posgrados académicos y en el área de la profesión para los posgrados profesionalizantes. Se entiende que en un país con una oferta limitada de estudios doctorales, uno en la UNAH, dos en la U PN FM y uno en una universidad privada, se busque gar antizar el acceso de los graduados de "cualquier" maestría a estudios doctorales, pero en este nivel se repite lo que se da a nivel de maestría, los estudiantes no saben cómo hacer "un aporte novedoso al conocimiento y la ciencia", léase no saben hacer investigación básica y de allí que la eficiencia terminal para ese nivel sea aún menor que el promedio de las maestrías.

\section{Resultados}

En base a lo planteado hasta el momento, parece ser que la diferencia principal entre ambos tipos de investigación es la clave para poder diferenciar los programas de posgrado. La investigación básica o pura 
tiene como finalidad la obtención y recopilación de información para ir construyendo una base de conocimiento que se va agregando a la información previa existente. La investigación aplicada, por su parte, tienen co mo objetivo resolver undeterminado problema o planteamiento específico. Es así que la investigación básica tiene en el laboratorio uno de sus campos de mayor desarrollo dado que permite el control de variables necesario para el establecimiento de relaciones causales, sin embargo, este hecho de que sea realizada "fuera de contexto" limita la transferencia de los hallazgos a las condiciones naturales de dichos fenómenos, es decir, limita su aplicabilidad a la solución de problemas, cosa que si hace la investigación aplicada. (Gutiérrez, 2010) .

De acuerdo a lo anteriormente planteado se puede establecer una diferenciación de la siguiente manera:

\section{Cuadro 1}

Diferencias entre posgrados académicos y profesionalizante ${ }^{1}$

\begin{tabular}{|c|c|}
\hline Posgrado Académico & Posgrado Profesionalizante \\
\hline $\begin{array}{l}\text { Es un proceso de formación cuya } \\
\text { naturaleza es esencialmente de } \\
\text { investigación básica. Concluye con } \\
\text { una tesis como requisito de } \\
\text { graduación el cual consiste en } \\
\text { investigación o creación intelectual, } \\
\text { nuevo conocimiento, requiere una } \\
\text { perspectiva epistemo lógica conceptual } \\
\text { y una propuesta meto dológica. Da } \\
\text { acceso a estudios de doctorado. }\end{array}$ & $\begin{array}{l}\text { Es un proceso de formación cuya } \\
\text { naturaleza es esencialmente de } \\
\text { investigación aplicada. Se } \\
\text { entenderá como un proceso de } \\
\text { formación teórico-práctico que } \\
\text { incorpora elementos investigativos y } \\
\text { desarrolla competencias profesionales } \\
\text { que son de utilidad en el trabajo diario } \\
\text { de los estudiantes. Concluye con un } \\
\text { trabajo final de graduación teórico } \\
\text { práctico que incluye modelos o } \\
\text { sistemas de intervención o } \\
\text { tratamiento de la realidad. No da } \\
\text { acceso a estudios de doctorado? }\end{array}$ \\
\hline
\end{tabular}

\footnotetext{
${ }^{1}$ Fuente DICyP 2014

${ }^{2}$ En la actualidad existen universidades que ofrecen Doctorados Profesionalizantes para quienes estudiaron una maestría profesionalizantes.
} 
En el cuadro anterior surgieron otros dos elementos diferenciadores, además del tipo de investigación que se realiza en cada tipo de programa: El trabajo final de graduación y si brinda 0 no acceso a continuar estudios doctorales. Estos temas se abordan en detalle en los apartados siguientes.

A hora bien, la diferencia fundamental no está dada por los elementos antes mencionados, sino por los propósitos de cada tipo de programa, las maestrías académicas buscan formar investigadores y las profesionalizantes, como su nombre lo indica, busca formar profesionales especialistas en su área. Como ejemplo, un graduado de una maestría en matemáticas de la UPN FM estará listo para la docencia en esa área, en tanto que un estudiante de una maestría en Derecho Marítimo de la UNAH estará preparado para litigar en aspectos relacionados con la gente de mar, respeto a convenios internacionales, manejo y desecho de lastres, entre otras. No se espera de ninguno de ellos que realicen investigación que permita ensanchar la frontera del cono cimiento en sus áreas, ni probar ni desarrollar teorías. Se espera que sean profesionales capaces y manejen los conocimientos y tengan las competencias propias de su área en el nivel de postgrado.

\section{Cuadro 2}

0 bjetivos según tipo de posgrado

\begin{tabular}{|l|l|}
\hline \multicolumn{1}{|c|}{ MaestríaAcadémica } & \multicolumn{1}{|c|}{ Maestría Profesionalizante } \\
\hline $\begin{array}{l}\text { Formar investigadores y docentes de } \\
\text { alto nivel competitivo, capaces de } \\
\text { contribuir al desarrollo humano }\end{array}$ & $\begin{array}{l}\text { Formar profesionales y docentes } \\
\text { capaces de proponer alternativas de } \\
\text { solución a problemas específicos de } \\
\text { sostenible, la integración regional, con } \\
\text { enfo que en la gener ación de } \\
\text { conompo de especialización conforme } \\
\text { a los requerimientos del desarrollo } \\
\text { científica, la constiante la investigación } \\
\text { científico, tecno lógico, político, } \\
\text { con El Estado, la sociedad y los sectores } \\
\text { productivos en alineamiento con los } \\
\text { objetivos de desarrollo nacional, social y cultural de la } \\
\text { científico y tecnológico. }\end{array}$ \\
\hline
\end{tabular}

Fuente: DIC yP, 2014 
En este sentido entonces, aparece como central la diferenciación entre posgrados académicos y profesionales. La discusión implica diversos aspectos aso ciados a la conceptualización, los objetivos, la orientación, las características, el papel de la investigación, los criterios de calidad, entre otros.Y que puede resumirse en la necesidad de que a nivel de las políticas de posgrado se pueda conceptualizar las características diferenciales entre los posgrados con orientación a la investigación como práctica académica y aquello s orientados al desar rollo profesional. (Dávila, 2011)

\section{La Investigación en los Planes de Estudio de los Posgrados}

A pesar de que existe una marcada diferencia entre el tipo de investigación que se realiza en los diferentes programas de posgrado, como se explicó anteriormente en el país esta diferencia no se da y todos los posgrados deben culminar con una tesis de formato académico. Esto lleva a que se introduzca el componente de investigación en los planes de estudio como asignaturas o talleres que buscan fortalecer las competencias de los estudiantes en el área de investigación, sin embargo al no tener claridad sobre lo que se debe saber y lograr con la investigación en cada postgrado, los espacios pedagógicos están determinados antojadizamente, es decir, existen posgrados que cuentan hasta con cinco espacios dedicados a investigación en sus distintas modalidades (Talleres, seminarios, etc. Ver Gráfico 1) y existen posgrados (más del $27 \%$ ) en los que el componente de investigación es igual o menor al 10 por ciento de las asignaturas.

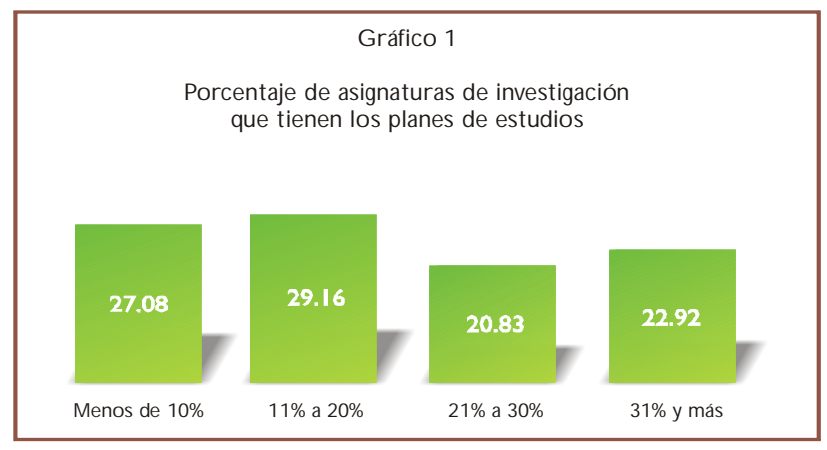

Paradigma - Revista de Investigación Educativa. Año 21. No. 35 
$\mathrm{N}$ ormativamente hay un requerimiento de incluir la investigación en los planes de estudio, la Dirección de Educación Superior (1993) establece que en el diseño de los planes de estudio se tiene que incorporar cursos que brinden las bases teórico-prácticas para desarrollar su trabajo de tesis. Lo que sugiere que en el diseño de los planes se debe contemplar realizar la formación que brinde el entrenamiento necesario para realizar investigaciones. $\mathrm{N}$ o obstante la normativa no es precisa para establecer en qué momento y cuánto contenido es necesario para cubrir este requisito, teniendo en cuenta que se trata de programas de postgrado la expectativa de la formación en investigación se vuelve alta.

Teniendo en cuenta además que es una exigencia la realización de un trabajo de investigación como requisito de graduación. Se podría pensar anticipadamente que quienes tienen mayor cantidad de asignaturas 0 espacios dedicados a la investigación lograrán una mayor eficiencia terminal, sin embargo en la práctica esto no es siempre el caso.

Gráfico 2

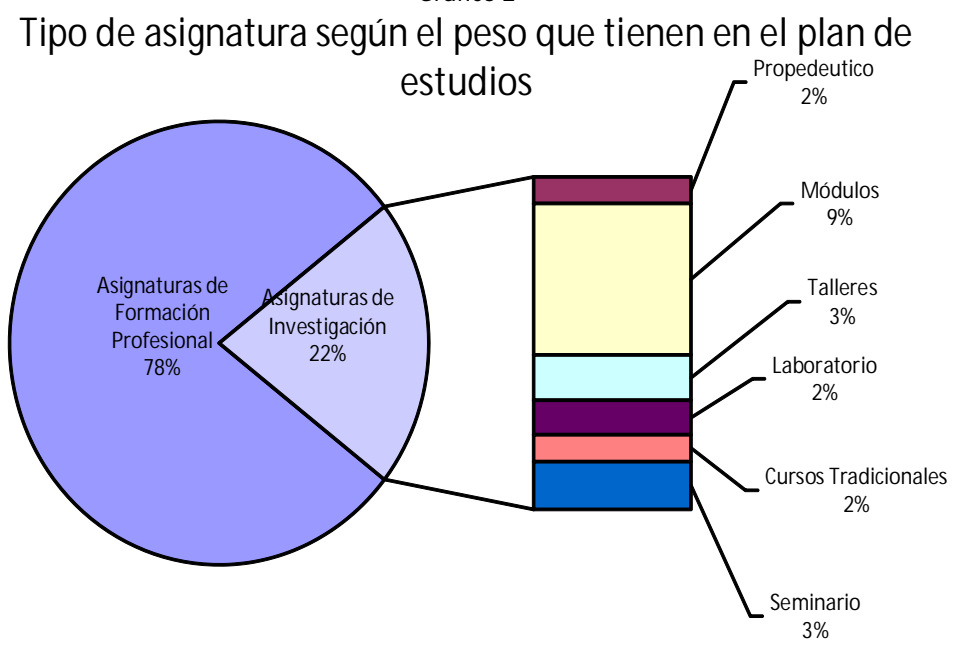

En la gráfica anterior se puede apreciar que las asignaturas de formación profesional ocupan más de tres cuartos de las asignaturas que se cursan en un programa de posgrado, y que las asignaturas de investigación se 
imparten de manera tradicional a través de módulos, talleres y seminarios. Esto es una muestra más del énfasis (orientación) de los posgrados en el país, donde se aprecia la inclinación hacia la profesionalización y no hacia la investigación. La investigación en todo caso se enseña como una asignatura más y no como una práctica académica, es decir, los estudiantes no están en los laboratorios 0 haciendo pasantías, o realizando investigaciones, o participando en congresos de investigación o escribiendo y discutiendo papers.

Visto de esta manera (Grafica 3) pareciera que se está formando profesionales que tienen que hacer una tesis académica para la cual no les preparan las asignaturas de investigación, que en más del 75 por ciento de los casos tiene un 30 por ciento o menos en este componente. Puesto de otra manera y utilizando los mismos ejemplos de la maestría en enseñanza de las matemáticas (U PN FM) y de la maestría en Derecho Marítimo (UNAH), equivaldría a pedirle al docente de matemáticas y al profesional del derecho que en lugar de dar clases de matemáticas y de litigar sobre derecho marítimo, se dediquen a investigar sin que el sistema educativo - no solo el posgrado- lo s haya preparado para ser investigadores. (Ver Cuadro 3).

\section{Cuadro 3}

Contenidos de Investigación en los planes de estudio

\begin{tabular}{|c|l|}
\hline \multicolumn{2}{|c|}{$\begin{array}{c}\text { Cantidad de contenidos e investigación que tienen los planes de } \\
\text { estudios según carrera de postgrado }\end{array}$} \\
\hline Rangos porcentuales & Carreras de postgrado \\
\hline Menos de 10\% & $\begin{array}{l}\text { Contaduría, O rdenamiento y Gestión delTerritorio, } \\
\text { Ingeniería de la Construcción y G erencia de } \\
\text { Proyectos, Maestría en Salud Pública, Esp. en } \\
\text { Oncología Q uirúrgica, Esp. en O torrino y Cirugía } \\
\text { de Cabeza y Cuello, Esp. en Psicología, D erecho } \\
\text { Penal y Procesal Penal, Liter atura a } \\
\text { Centroamericana, Demartología, Cirugía G eneral, } \\
\text { Cirugía Plástica, Anatomía Patólogica }\end{array}$ \\
\hline
\end{tabular}




\begin{tabular}{|c|c|}
\hline $11 \%$ a $20 \%$ & $\begin{array}{l}\text { Mercadotécnia con Énfasis en N egocios } \\
\text { Internacionales, Postg. en Traumatología y } \\
\text { O rtopedía, Esp. en Pediatría, Derecho Mercantil, } \\
\text { Derechos Humanos y Desarrollo, D iseño, Gestión } \\
\text { y Evaluación C urricular, D ocencia Superior, } \\
\text { C iencias Políticas y G estión Estatal, A nestesiología, } \\
\text { Economía y Planificación en D erecho, Planificación } \\
\text { y D esarrollo Turístico, Formulación, Gestión y } \\
\text { Evaluación de Proyectos, Gestión Social Urbana. }\end{array}$ \\
\hline 21\%а30\% & $\begin{array}{l}\text { Epidemilogía, Planeación y D esar rollo, } \\
\text { Comunicación y Tecnologías Educativas, Gestión } \\
\text { de Empresas Cooperativas, D irección de N egocios } \\
\text { Internacionales, Gestión de Riesgos y Manejo de } \\
\text { D esastres,A ctividad Física para la Salud, Postg. en } \\
\text { Med. de Rehabilitación, Forestaría Comunitaria, } \\
\text { Ciencias Sociales con O rientación en G estión del } \\
\text { D esarrollol. }\end{array}$ \\
\hline 31\%y más & $\begin{array}{l}\text { Medicina del Trabajo, Enfermería en Salud Fami- } \\
\text { liar, Enfermería en Salud Materno Perinatal, } \\
\text { O ftalmología, Enfermería en A tención en Salud } \\
\text { Integral del N iño y Adolescente, Educación Supe- } \\
\text { rior, Literatura Centroamericana, Enfermería de } \\
\text { Q uirófanos, Maestría Latinoamericana en Trabajo } \\
\text { Social, D emo grafía Social, Especialidad en } \\
\text { Desarrollo Humano y Cooperación Local e } \\
\text { Internacional. }\end{array}$ \\
\hline
\end{tabular}

Fuente: Moncada, 2011

Al parecer algunos posgrados tendrían ventajas comparativas al momento de realizar una tesis académica y otros tendrían mayores dificultades, sin embargo como se mencionó anteriormente los posgrados de medicina (Figura 1) tienen la mejor eficiencia terminal de la UNAH y apenas llevan una asignatura relacionada con investigación, en tanto Trabajo Social y desarrollo (Figura 2) tiene más de 8 asignaturas relacionadas con investigación. $\mathrm{H}$ ay diferencias entre el tipo de trabajo que se presenta en cada una, eso lo abordaremos próximamente, pero el programa deTrabajo So cial y Desarrollo no se 
acerca siquiera a la eficiencia de las especialidades médicas, lo que implica que no basta con "incluir" asignaturas de investigación en el currículo de los programas. (Ver figuras 1 y 2)

Figura 1. Flujograma especialidad Médica Figura 2. Flujograma Maestría

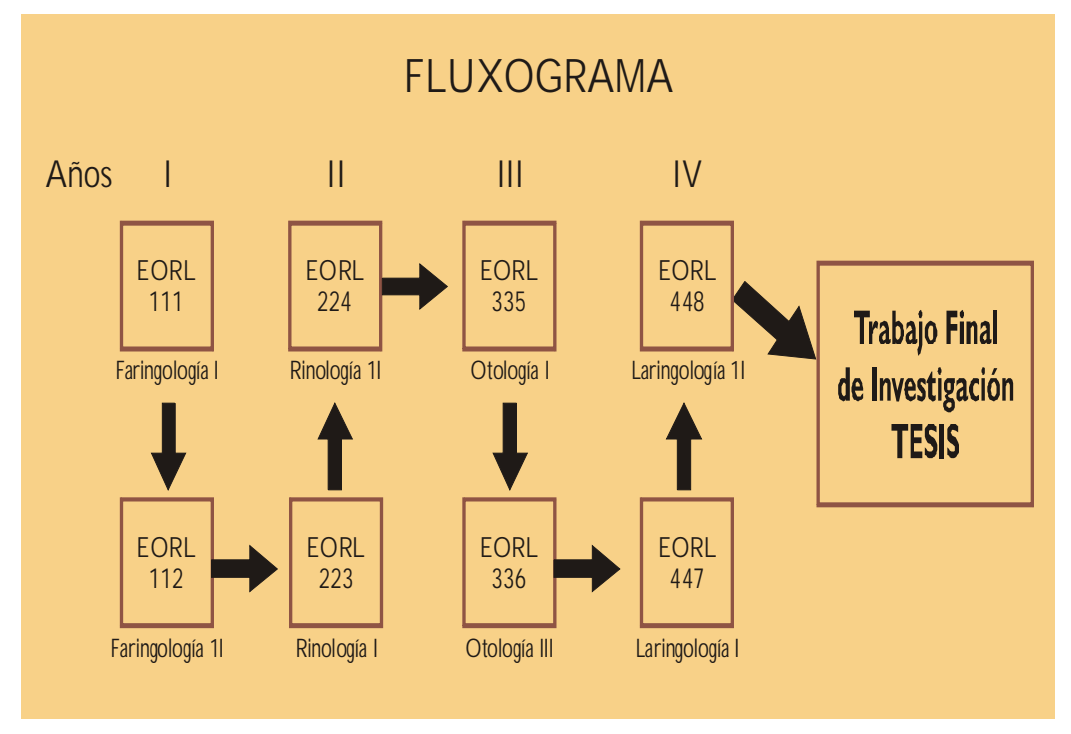

\section{Flujograma}

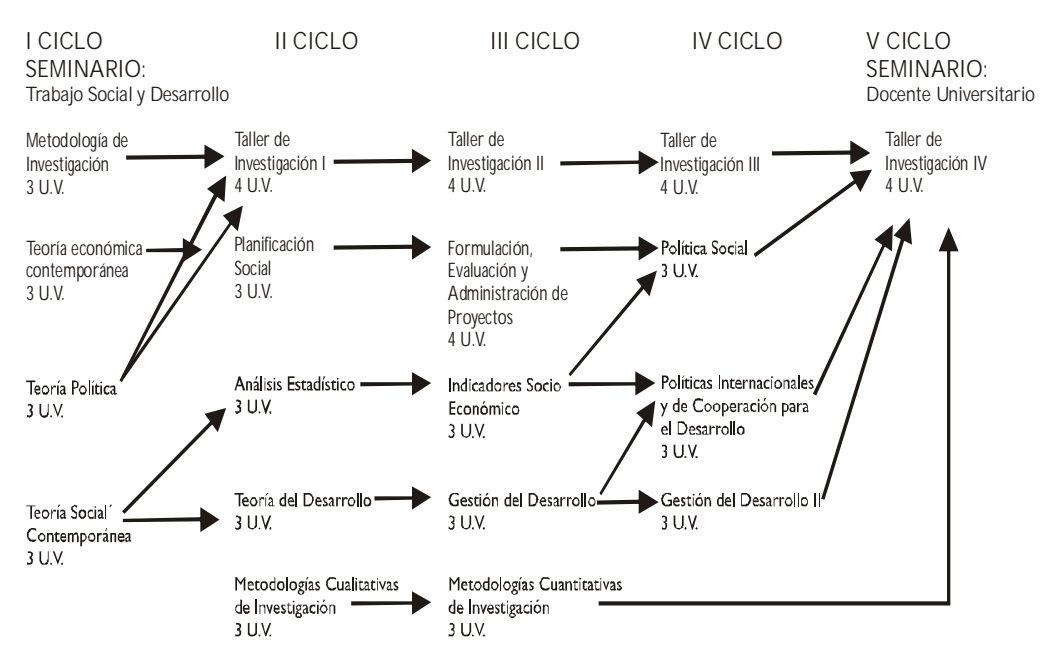

Paradigma - Revista de Investigación Educativa. Año 21. No. 35 


\section{Conclusiones}

Uno de los principales problemas que se tienen al no diferenciar ambos tipos de posgrado, es que la orientación de un posgrado para el trabajo académico termina considerándose equivalente a la formación para el trabajo profesional por tanto, ambos se desarrollan y evalúan de manera similar, es decir académica. D e esta manera, se establecen criterios de calidad basados en aspectos relacionados con la aplicación adecuada del proceso de investigación básica, forzando en muchos casos a los estudiantes a seguir ciegamente un modelo de investigación que por lo general no se ajusta a la realidad de la problemática profesional y por ende no aporta respuestas a su solución.

A quí surge otro problema, el relacionado con delimitar rigurosamente ambos tipos de posgrado y establecer una suerte de frontera que no permita que uno y otro se relacionen sino que los presenta como antagónicos. Hay áreas como las vinculadas a las ciencias aplicadas, ingenierías y demás carreras tecnológicas, en la que en muchas ocasiones se deben incluir procesos de investigación en los currículos - sin embargo este parece no ser el caso de la maestría en Ingeniería de la Construcción y Gerencia de Proyectos en la que el currículo no contempla espacios de investigación- también puede darse el caso opuesto, en el que un posgrado académico deba incluir prácticas y contenidos más profesio nalizantes que directamente relacio nados con procesos de investigación. En estos casos, según Dávila (2011) los conceptos de académico y profesional serían más complementarios que contradictorios, partes de un continuo asociado a las formas de producción del conocimiento en esas áreas.

Se concluye, tal como lo plantea D ávila (2011) que "existe la necesidad de readecuar las políticas en el nivel de posgrado, de manera que reconozcan las diferencias entre ambos tipos de posgrados y contemplen las necesidades difer enciales de cada uno. En ambos casos se vuelve necesaria la configuración de un sistema de posgrado en constante actualización y desar rollo que dé respuesta a las necesidades de la sociedad." El reto es claro para que los nuevos posgrados se definan en función de sus objetivos, su metodología, su currículo, los trabajos finales, el campo profesional y la función que la investigación científica desempeña dentro del mismo. Pero además, el reto se prolonga hacia los posgrados existentes para que a través de los procesos de autoevaluación y procesos de acreditación puedan (re)definirse como profesionalizantes o académicos. 
La Investigación en los Posgrados Académicos y Profesionalizantes en Honduras

\section{Referencias Bibliográficas}

A maya, J. (2014) Balance de los Posgrados en H onduras, U PN FM, H onduras.

Dávila, M. (2011) Posgrados académicos y profesionales. La discusión actual en Argentina y Brasil. III Encuentro Internacional de Estudios Comparados en Educación, Buenos Aires,A rgentina.

Dirección de Educación Superior (1993) Ley de Educación Superior, Consejo de Educación Superior, Honduras.

D irección de Investigación C ientífica y Postgrado, (2014) Los posgrados académicos y Profesionalizantes. UN AH, Honduras.

Gutiérrez, G. (2010) Investigación Básica y aplicada en Psicología: Tres modelos de desarrollo. DIA LN ET, Colombia.

Moncada, German (2011) Estado actual de los postgrados de la UNAH, D irección de Investigación Científica, U N AH, Honduras.

Montemayor, M., García, M. y G arza,Y. (2002) Guía para la investigación documental, Trillas, México.

Rama, C . (2007) Los postgrados en América Latina y El Caribe en la sociedad del conocimiento, UDUAL, México.

Sánchez, M. (2008), Una propuesta conceptual para diferenciar los programas de postgrado profesionalizantes y orientados a la investigación. Implicaciones para la regulación, el diseño y la implementación de los programas de postgrado, Cienciay So ciedad, vol. XXXIII, núm. 3, JulioSeptiembre 2008, pp. 327-341, Instituto tecnológico de Santo D omingo, República Dominicana

UNAH, (2008/2014) Revista de Postgrado. Dirección de Postgrado. Honduras. 
Edwin Roldán M edina López

UPN FM (2014) Oferta de Posgrados descargado de la página web: www.upnfm.edu.hn recuperado el 3 de noviembre de 2014. 